\title{
TREM-1 expression in craniopharyngioma and Rathke's cleft cyst: its possible implication for controversial pathology
}

\author{
Yi Liu ${ }^{1, *}$, Chao-hu Wang ${ }^{1, *}$, Dan-ling Li ${ }^{2, *}$, Shi-chao Zhang ${ }^{1}$, Yu-ping Peng ${ }^{1}$, Jun- \\ xiang Peng ${ }^{1}$, Ye Song ${ }^{1}$, Song-tao $Q i^{1}$, Jun Pan ${ }^{1}$ \\ ${ }^{1}$ Department of Neurosurgery, Nanfang Hospital of Southern Medical University, Guangzhou, China \\ ${ }^{2}$ Department of Biometric, College of Public Health of Southern Medical University, Guangzhou, Guangdong, China \\ *These authors contributed equally to this work \\ Correspondence to: Jun Pan, email: panjundu@hotmail.com \\ Song-tao Qi, email: qisongtaosjwk@163.com
}

Keywords: craniopharyngioma, metaplasia, Rathke's cleft cyst, SE, TREM- 1

Received: March 10, 2016

Accepted: June 30, 2016

Published: July 8, 2016

\section{ABSTRACT}

\begin{abstract}
Whether a mixed type of craniopharyngioma (CP) exists and whether papillary craniopharyngioma (pCP) is on a histopathological continuum with Rathke's cleft cyst (RCC) remain controversial. Herein, we examined the expression and localization of $\beta$-catenin, BRAF p.V600E (V600E), and triggering receptor expressed on myeloid cells-1 (TREM-1) in 58 samples including 20 pCPs, 26 adamantinomatous craniopharyngiomas (aCP), and 12 RCCs. Five aCPs were diagnosed with mixed type CPs and the remaining 21 cases were pure aCPs. Four of the 12 RCCs presented with significant squamous epithelium (SE). V600E immunoreactivity was observed in all pCPs in the cytoplasm, but not in the nuclei. aCPs and RCCs, including mixed type CP, did not express V600E. Nuclear $\beta$-catenin translocation was detected exclusively in aCPs. TREM-1 was expressed in PCPs. Additionally, TREM-1 expression was detected in the SE of 5 "mixed type" CPs, while it was absent in pure aCPs. TREM-1 was expressed in 4 RCCs with SE, but not in the remaining 8 RCCs. TREM-1 mRNA levels were compared in cultured PCP and aCP cells. TREM-1 MRNA level was significantly ( $p<0.001$; up to 4.045 fold) higher in pCPs than in aCPs. Western blotting revealed a significantly ( $p<0.001$; up to 7.19 fold) lower level of TREM-1 expression in aCP cells compared to that in PCP cells. Our findings further supported that RCC and PCP may represent two ends of a morphological spectrum. A variant showing overlapping histological features of aCP and PCP should not be considered as a mixed type.
\end{abstract}

\section{INTRODUCTION}

Craniopharyngiomas (CPs) are complex epithelial neoplasms of the sellar region arising along the site of the vestigial craniopharyngeal tract. In their pure form, adamantinomatous craniopharyngiomas $(\mathrm{aCP})$ and papillary craniopharyngioma $(\mathrm{pCP})$ are clinicopathologically distinct [1-4]. The observation of some overlapping features, mainly the squamous epithelium (SE) component, between aCP and $\mathrm{pCP}$ led to the hypothesis that a mixed type may exist [5-8]. Previous reports suggest that a mixed type presenting with the characteristic histopathologic findings of aCP and $\mathrm{pCP}$ exists. Additionally, there are frequent admixtures of both squamous and adamantinomatous areas within the same tumor, which were only identified by conventional microscopy without immunohistochemistry. pCP's resemblance with Rathke's cleft cyst (RCC) with SE also supports the hypothesis that $\mathrm{pCP}$ is on a histopathological continuum with RCC [9]. However, these two hypotheses remained controversial. Some researchers consider that SE exists in $\mathrm{aCP}$ and $\mathrm{RCC}$ as a result of squamous metaplasia mediated by inflammation $[10,11]$.

Genetic and molecular pathologic features support a distinct pathogenesis of aCP and $\mathrm{pCP}$ [12-17]. It was reported that $\mathrm{pCPs}$ present BRAF p.V600E mutations in $95 \%$ of cases and CTNNB1 mutations are exclusive and specific to aCPs [16]. The triggering receptor expressed 
on myeloid cells-1 (TREM-1) is a cell surface receptor and a member of the immunoglobulin superfamily that potently amplifies inflammatory responses by inducing the secretion of pro-inflammatory mediators. TREM-1 is known as an activating receptor expressed on neutrophils, monocytes, and macrophages $[18,19]$. Limited data are available on the role of TREM-1 in chronic inflammation and tumorigenesis. Previous studies $[10,11]$ and our studies $[20,21]$ showed that inflammation is common in CP and RCC. Since TREM-1 plays a significant role in the inflammatory response, whether TREM-1 is expressed in $\mathrm{CP}$ and RCC should be further studied.

The present study was designed to clarify two problems by using these biomarkers: one is whether aCP with $\mathrm{SE}$ represents a mixed type of $\mathrm{CP}$; the second is whether RCC with SE is a precursor of $\mathrm{pCP}$.

\section{RESULTS}

\section{Patient characteristics}

Thirty three male and 25 female patients with a mean age of 37 years (range 7-61 years) were enrolled in this study. Fifty-eight samples, including $20 \mathrm{pCPs}$, 26 aCPs, and 12 RCCs, were obtained and used for analysis. Five aCP cases were diagnosed with mixed type $\mathrm{CP}$ according to previous studies $[7,8]$ and the remaining 21 cases were pure aCPs. Four of the 12 RCCs presented with significant SE. In order to eliminate the influence of mesenchymal cells in tumor tissues, we used both tumor tissues and cultured CP cells in this study. All CP samples were successfully cultured. We used the anchorage velocity-dependent separation method to exclude mesenchymal cells and passage 3-cells were used for experiments. Cultured CP cells were identified by assessing the expression of pan-CK. We also identified the cells by immunostaining using BRAF V600E and $\beta$-catenin antibodies (Figure 1).

\section{Differential distribution pattern of BRAF V600E in CPs and RCCs tissues}

We examined the immunohistochemical distribution pattern of BRAF V600E (V600E) in all specimens. V600E immunoreactivity was strongly detected in the SE of all pCPs in the cytoplasm, but the immunoreactivity was not detected in the nuclei. V600E immunoreactivity was absent in all aCPs (including "mixed type CP") and RCCs (including those with SE specimens) (Figure 2).

\section{Differential distribution pattern of $\beta$-catenin in CP and RCC tissues}

Nuclear $\beta$-catenin translocation was exclusively detected in all aCPs (including "mixed type CP"), especially in epithelial nodules. $\beta$-catenin remained at the cell membrane in pCPs and RCCs (Figure 2).

\section{Differential distribution pattern of TREM-1 in CP and RCC tissues}

TREM-1 immunoreactivity was observed in the SE of all pCPs with strong detection in the cytoplasm and nuclei. Immunoreactivity was also detected in the SE of aCPs (mixed type CP), while absent in aCPs without SE (pure aCPs). TREM-1 was expressed in 4 RCC with SE, mainly in the cytoplasm and nuclei of the SE in RCC. The remaining 8 RCCs without SE did not express TREM-1 (Figure 2).

\section{Mutation distributions}

\section{BRAF mutation}

BRAF mutation was detected in all pCP patients. All of them presented with the V600E mutation. In patients with aCP, including 21 pure aCPs and 5 "mixed type" CPs, BRAF mutation was absent. All patients with RCC were also negative for BRAF mutation.

\section{CTNNB1 exon 3 mutation}

CTNNB1 exon 3 mutation was detected in all patients with aCP, including 21 pure aCPs and 5 "mixed type" CPs, while the mutation was absent in all patients with pCP and RCC. We found 5 mutational sites. The mutational analysis of CTNNB1 exon 3 mutations in patients with aCP is summarized in Table 1. More than $50 \%$ of the mutations $(14 / 26)$ were at the "hotspot" Ser and $\mathrm{Thr}$ residues.

\section{TREM-1 expression in CP cells}

We compared TREM-1 expression in aCP and pCP cells using double-immunofluorescence staining (Figure 3). TREM-1 mRNA levels of aCP and pCP cells were compared by quantitative reverse transcription-polymerase chain reaction (qRT-PCR) from a collection of native tumor samples comprising $6 \mathrm{pCP}$ and $6 \mathrm{aCP}$ cultured cells. The evaluation of the relative TREM-1 expression revealed a significantly ( $\mathrm{p}<0.001$; up to 4.045 fold) higher level in pCP cells (mean $=5.66 \pm 0.39)$ than in aCP cells (mean $=1.40 \pm 0.25)$. Western blotting was employed to assess TREM-1 protein level in the same CP cultured cells. TREM-1 expression levels were significantly $(\mathrm{p}<$ 0.001 ; up to 7.19 fold) lower in aCP cells (mean $=0.13 \pm$ 0.07 ) than in pCP cells (mean $=0.97 \pm 0.03)$ (Figure 3 ).

\section{TREM-1 expression in CP tissues}

TREM-1 levels in aCP and pCP tissues were also compared by western blotting and qPCR from the same $6 \mathrm{pCP}$ and $6 \mathrm{aCP}$ samples. Unfortunately, one $\mathrm{aCP}$ and one pCP sample were too small for western blotting. 
Thus, only 5 aCP and 5 pCP samples were used. TREM1 expression levels were significantly $(\mathrm{p}=0.0228$; up to 1.89 fold) lower in aCP tissues (mean $=0.714 \pm 0.112$ ) than that in pCP tissues (mean $=1.453 \pm 0.238)$, as seen by western blotting. TREM-1 mRNA levels of the same $5 \mathrm{aCP}$ and 5 pCP tissues samples were compared by qRT-PCR. TREM-1 expression levels were significantly $(\mathrm{p}<0.001$; up to 2.08 fold $)$ higher in pCP tissues $($ mean $=2.99 \pm 0.12)$ than in aCP tissues $($ mean $=1.28 \pm 0.11)$ (Figure 4$)$.

\section{Differential distribution pattern of TREM-1 in SE of other tissues}

In order to examine the significance of TREM-1 expression in distinguishing normal SE from metaplastic SE, we examined TREM-1 expression in various tissues, including cervical SE and metaplastic SE from cervicitis. TREM-1 expression was strongly detected in the
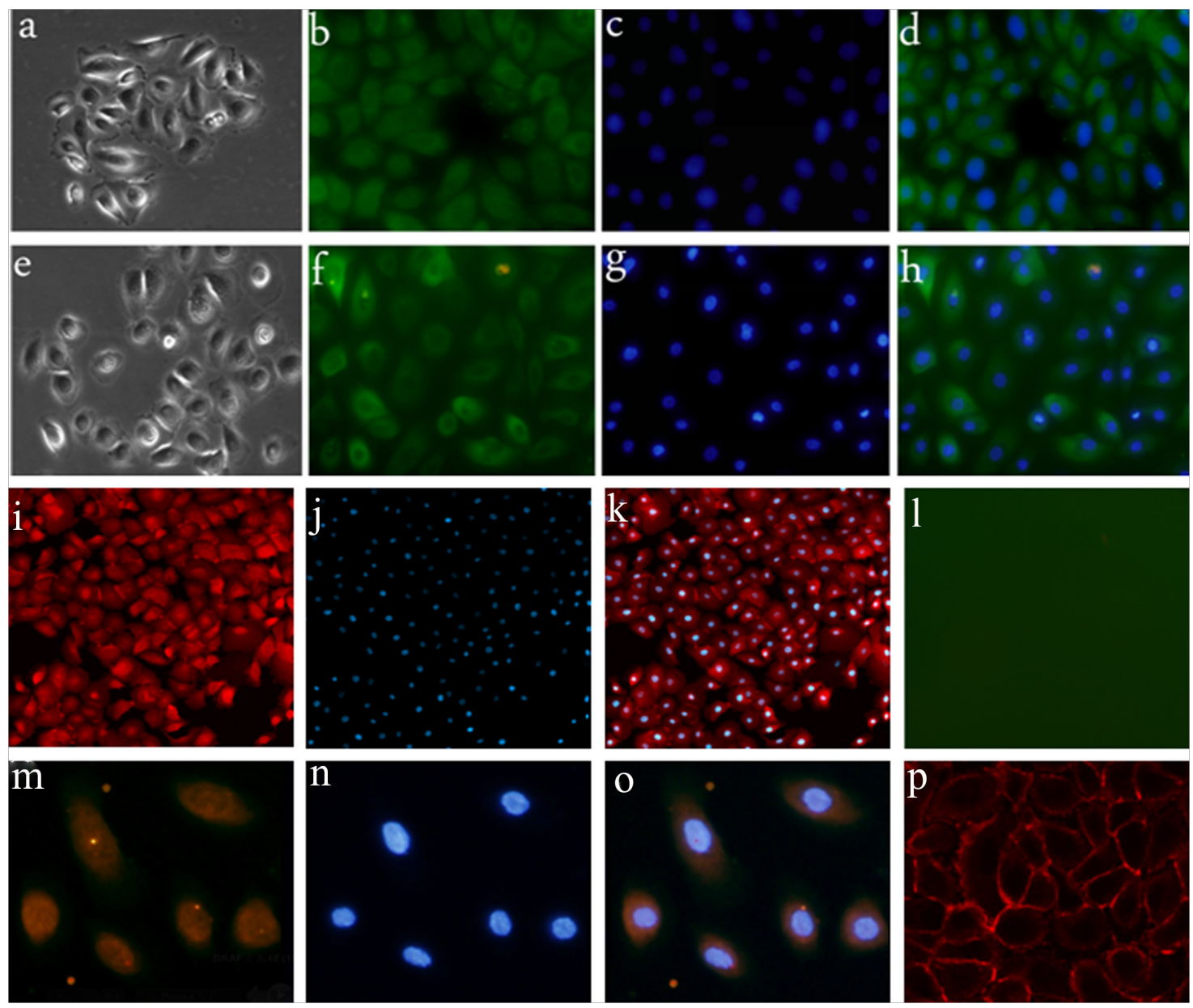

Figure 1: Primary cell culture of CP samples. a-d. Primary cell culture of aCP; a. morphology of aCP cells; b-d. aCP cells are positive for pan-CK. $(400 \times)$ e-h. Primary cell culture of pCP; e. morphology of pCP cells; f-h. pCP cells are positive for pan-CK.(400×) i-l. $\beta$-catenin and V600E expression in cultured aCP cells; i-k. nuclear $\beta$-catenin translocation is detected in cultured aCP cells; 1 . V600E immunoreactivity is absent in cultured aCP cells. $(400 \times)$ m-p. $\beta$-catenin and V600E expression in cultured pCP cells; m-o. V600E immunoreactivity is observed in cultured $\mathrm{pCP}$ cells with strong detection in the cytoplasm; $\mathrm{p}$. $\beta$-catenin is restricted to the cell membrane of cultured pCP cells. $(400 \times)$ 
A significant confusion may exist between the diagnosis of RCC and craniopharyngioma given that there may be overlapping histological features. Rathke's cleft cysts are classically lined by a simple cuboidal or columnar epithelium. Some RCCs appear to be a smooth transition from ciliated columnar epithelium to ciliated SE, to the stratified SE of craniopharyngiomas, which led to the hypothesis that the basal cells of RCC may transform to papillary-type craniopharyngiomas following squamous metaplasia. However, other researchers attributed focal squamous metaplasia in RCC to chronic inflammation in the cyst walls $[4,9]$.

The BRAF V600E mutation was recently described as a genetic hallmark of papillary craniopharyngiomas. Buslei et al. analyzed the BRAF mutational status of 33
Rathke's cleft cyst and 18 papillary craniopharyngioma specimens by immunohistochemistry using a monoclonal antibody (V600E) that selectively recognizes the BRAF V600E mutant epitope and additional BRAF pyrosequencing. All control cases of papillary craniopharyngiomas showed evidence of BRAF V600E mutation either by immunohistochemistry or by pyrosequencing. Three out of 33 cases with Rathke's cleft cysts showed genetic BRAF V600E alterations. Histological re-evaluation indicated that two of three positive cases were papillary craniopharyngiomas and the third case presented with unclear histology [22]. Our results were similar. In fact, immunohistochemistry indicated that SE from RCC was also BRAF V600E

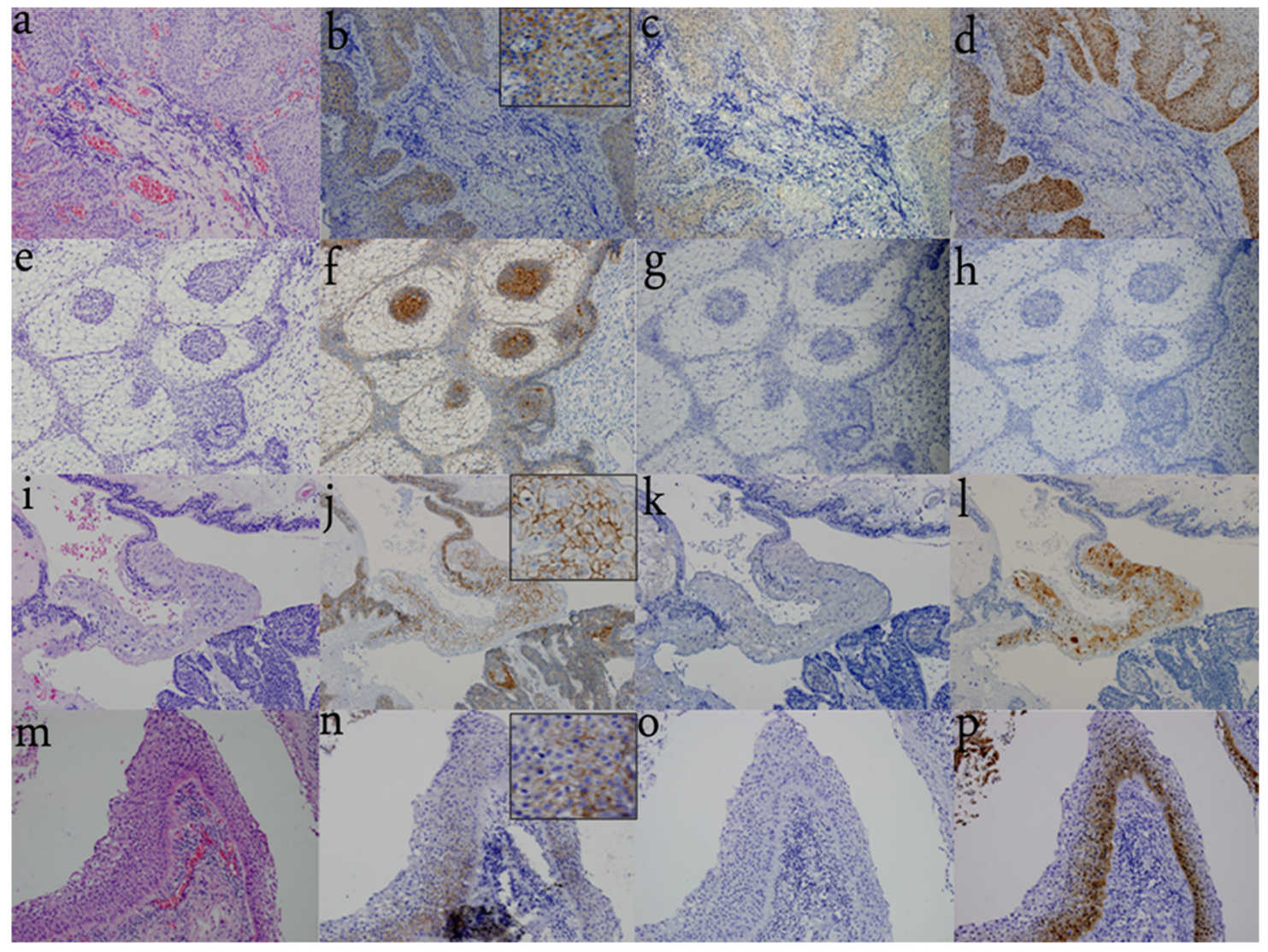

Figure 2: Differential distribution pattern of $\boldsymbol{\beta}$-catenin, V600E, and TREM-1 in CPs and RCCs. a. H\&E staining of pCP indicates the squamous and well-differentiated, non-keratinizing epithelium. $(200 \times)$ b. Nuclear $\beta$-catenin translocation is not observed and $\beta$-catenin expression is restricted to the cell membrane. $(200 \times)$ c. V600E immunoreactivity is observed in the SE of pCPs with strong detection in the cytoplasm, while it is not detected in the nuclei. $(200 \times)$ d. TREM-1 immunoreactivity is strongly detected in the cytoplasm and nuclei of the SE in all pCPs. $(200 \times)$ e. H\&E staining of pure aCP shows the classical appearance with peripheral palisading epithelium, loose 'stellate reticulum', and whorl-like cells. $(200 \times)$ f. $\beta$-catenin translocation is observed especially in whorl-like cells. $(200 \times)$ g. V600E immunoreactivity is not detected in pure aCP tissues. $(200 \times)$ h. TREM-1 immunoreactivity is also absent in pure aCP tissues. (200×) i. H\&E staining of "mixed type CPs" shows both SE and peripheral palisading epithelium, whorl-like cells are also detected. (200×) j. $\beta$-catenin translocation is observed in whorl-like cells and is restricted to the cell membrane in SE of "mixed type CP". (200×) k. V600E immunoreactivity is absent in SE and other components of "mixed type CP". (200×) l. TREM-1 immunoreactivity is observed in the SE of "mixed type CP" and absent in other components of "mixed type CPs". (200×) m. H\&E staining of RCC with stratified SE. (400×) n. No nuclear $\beta$-catenin translocation is observed and $\beta$-catenin expression is restricted to the cell membrane. $(400 \times) \mathbf{0}$. V600E immunoreactivity is absent in SE. $(400 \times)$ p. TREM-1 immunoreactivity is observed in the SE. $(400 \times)$ 
Table 1: Summary of the mutational analysis of CTNNB1 exon 3 in aCP

\begin{tabular}{|c|c|c|}
\hline & Histological diagnosis & mutational site \\
\hline 1 & Pure aCP & p.S37F $($ c. $110 \mathrm{C}>\mathrm{T})$ \\
\hline 2 & Pure aCP & p.D32G $($ c. $95 A>G)$ \\
\hline 3 & Pure aCP & p.S37F $($ c. $110 C>T)$ \\
\hline 4 & Pure aCP & p.S37F $($ c. $110 \mathrm{C}>\mathrm{T})$ \\
\hline 5 & Pure aCP & p.D32G $($ c. $95 A>G)$ \\
\hline 6 & Pure aCP & p.D32G $($ c. $95 A>G)$ \\
\hline 7 & Pure aCP & p.S37F $($ c. $110 \mathrm{C}>\mathrm{T})$ \\
\hline 8 & Pure aCP & p.T41I(c.122C $>$ T) \\
\hline 9 & Pure aCP & p.D32Y(c.94G $>$ T) \\
\hline 10 & Pure aCP & p.T41I (c.122C $>$ T $)$ \\
\hline 11 & Pure aCP & p.S37F $($ c. $110 \mathrm{C}>\mathrm{T})$ \\
\hline 12 & Pure aCP & p.D32G(c.95A>G) \\
\hline 13 & Pure aCP & p.D32Y(c.94G $>$ T) \\
\hline 14 & Pure aCP & p.D32G $($ c. $95 A>G)$ \\
\hline 15 & Pure aCP & p.D32Y(c.94G $>$ T) \\
\hline 16 & Pure aCP & p.D32G $($ c. $95 A>G)$ \\
\hline 17 & Pure aCP & p.S37C (c.110C $>$ G $)$ \\
\hline 18 & Pure aCP & p.S37F $(\mathrm{c} .110 \mathrm{C}>\mathrm{T})$ \\
\hline 19 & Pure aCP & p.T41I(c. $122 \mathrm{C}>\mathrm{T})$ \\
\hline 20 & Pure aCP & p.D32Y(c.94G $>$ T) \\
\hline 21 & Pure aCP & p.T41I (c.122C $>$ T $)$ \\
\hline 22 & Mixed CP & p.S37C $($ c. $110 C>G)$ \\
\hline 23 & Mixed CP & p.D32Y(c.94G $>$ T) \\
\hline
\end{tabular}

Nucleotide change

GGA ATC CAT TCT GGT GCC ACT > GGA ATC CAT TTT GGT GCC ACT

TCT TAC CTG GAC TCT GGA ATC > TCT TAC CTG GGC TCT GGA ATC

GGA ATC CAT TCT GGT GCC ACT > GGA ATC CAT TTT GGT GCC ACT

GGA ATC CAT TCT GGT GCC ACT > GGA ATC CAT TTT GGT GCC ACT

TCT TAC CTG GAC TCT GGA ATC > TCT TAC CTG GGC TCT GGA ATC

TCT TAC CTG GAC TCT GGA ATC > TCT TAC CTG GGC TCT GGA ATC

GGA ATC CAT TCT GGT GCC ACT > GGA ATC CAT TTT GGT GCC ACT

GGT GCC ACT ACC ACA GCT CCT > GGT GCC ACT ATC ACA GCT CCT

TCT TAC CTG GAC TCT GGA ATC > TCT TAC CTG TAC TCT GGA ATC

GGT GCC ACT ACC ACA GCT CCT > GGT GCC ACT ATC ACA GCT CCT

GGA ATC CAT TCT GGT GCC ACT > GGA ATC CAT TTT GGT GCC ACT

TCT TAC CTG GAC TCT GGA ATC > TCT TAC CTG GGC TCT GGA ATC

TCT TAC CTG GAC TCT GGA ATC > TCT TAC CTG TAC TCT GGA ATC

TCT TAC CTG GAC TCT GGA ATC > TCT TAC CTG GGC TCT GGA ATC

TCT TAC CTG GAC TCT GGA ATC > TCT TAC CTG TAC TCT GGA ATC

TCT TAC CTG GAC TCT GGA ATC > TCT TAC CTG GGC TCT GGA ATC

GGA ATC CAT TCT GGT GCC ACT > GGA ATC CAT TGT GGT GCC ACT

GGA ATC CAT TCT GGT GCC ACT > GGA ATC CAT TTT GGT GCC ACT

GGT GCC ACT ACC ACA GCT CCT > GGT GCC ACT ATC ACA GCT CCT

TCT TAC CTG GAC TCT GGA ATC > TCT TAC CTG TAC TCT GGA ATC

GGT GCC ACT ACC ACA GCT CCT > GGT GCC ACT ATC ACA GCT CCT

GGA ATC CAT TCT GGT GCC ACT > GGA ATC CAT TGT GGT GCC ACT

TCT TAC CTG GAC TCT GGA ATC > TCT TAC CTG TAC TCT GGA ATC

(continued) 


\begin{tabular}{|c|c|c|c|}
\hline & Histological diagnosis & mutational site & Nucleotide change \\
\hline 24 & Mixed CP & p.S37C (c. $110 \mathrm{C}>\mathrm{G})$ & $\begin{array}{c}\text { GGA ATC CAT TCT GGT GCC ACT }> \\
\text { GGA ATC CAT TGT GGT GCC ACT }\end{array}$ \\
\hline 25 & Mixed CP & p.T41I(c.122C>T) & $\begin{array}{c}\text { GGT GCC ACT ACC ACA GCT CCT > } \\
\text { GGT GCC ACT ATC ACA GCT CCT }\end{array}$ \\
\hline 26 & Mixed CP & p.D32Y(c.94G > T) & $\begin{array}{c}\text { TCT TAC CTG GAC TCT GGA ATC }> \\
\text { TCT TAC CTG TAC TCT GGA ATC }\end{array}$ \\
\hline
\end{tabular}

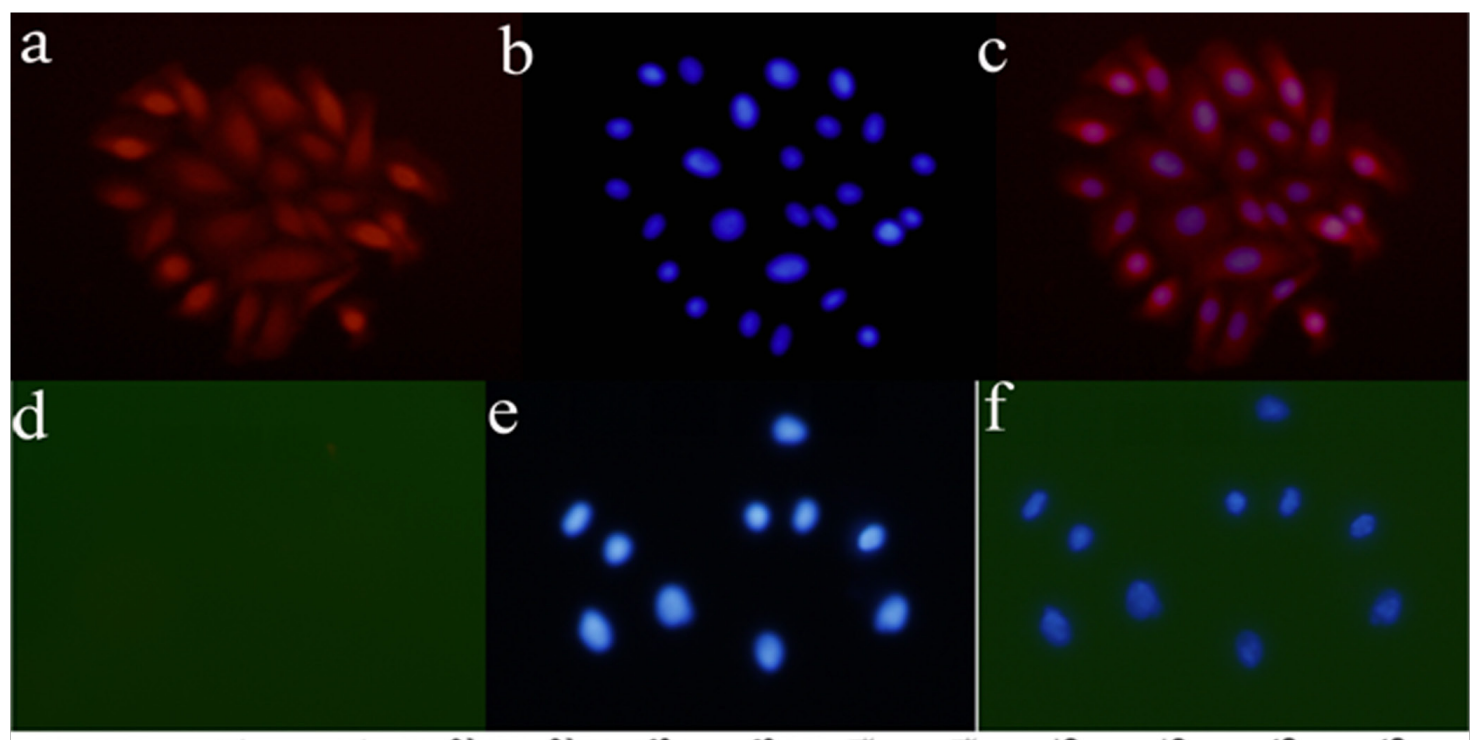

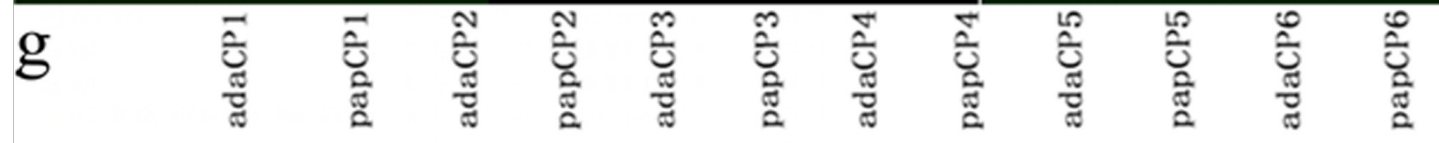
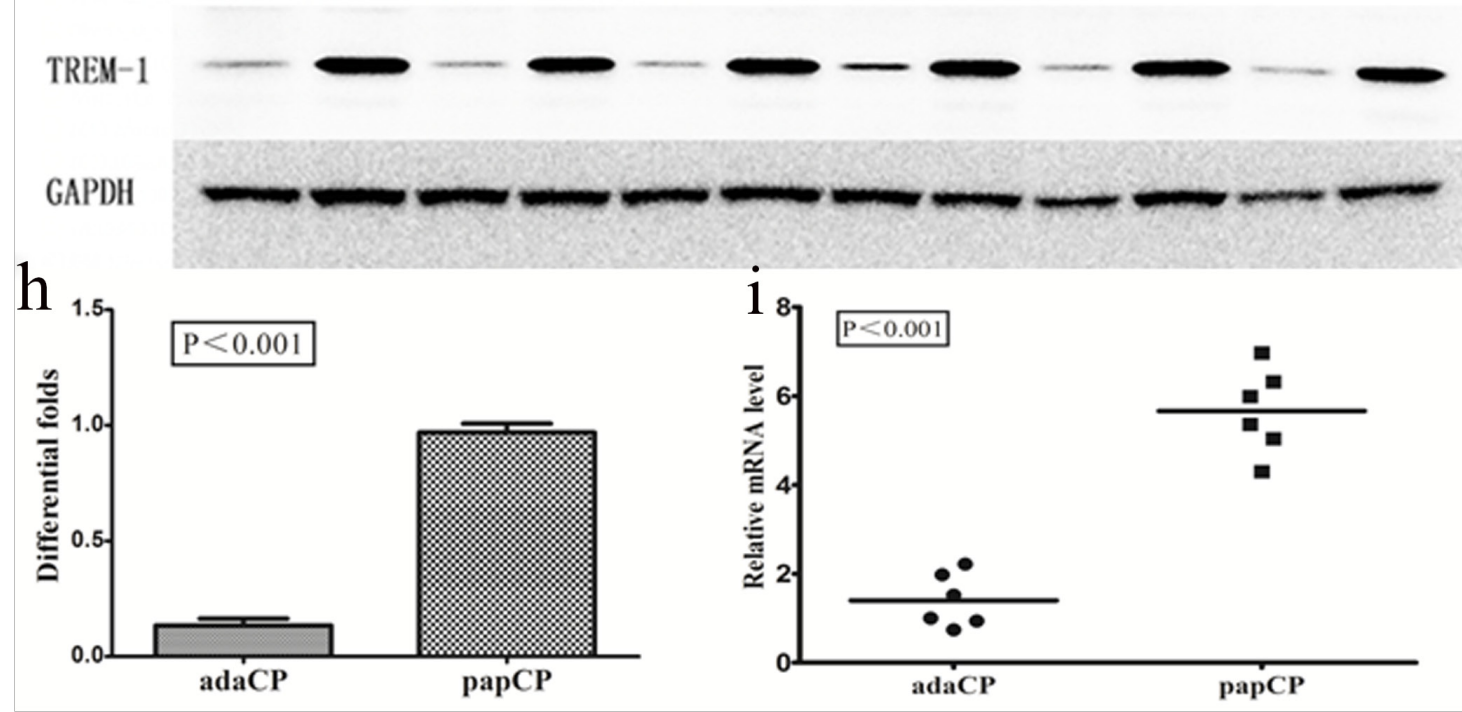

Figure 3: TREM-1 expression pattern in aCP and pCP cells. a-c. TREM-1 expression is strongly detected in the cytoplasm and nuclei of pCP cells. (400×) d-f. TREM-1 expression is absent in aCP cells. (400×) g-h. Western blotting shows the protein level of TREM-1 in cultured CP cells. Evaluation of the relative TREM-1 expression revealed a significantly ( $p<0.001$; up to 7.19 fold) lower level in $\mathrm{aCP}($ mean $=0.13 \pm 0.07)$ compared to that of the $\mathrm{pCP}$ variant (mean $=0.97 \pm 0.03) \mathrm{i}$. $T R E M-1 \mathrm{mRNA}$ levels of aCP and $\mathrm{pCP}$ cells were compared by qRT-PCR. TREM-1 relative expression level was significantly ( $\mathrm{p}<0.001$; up to 4.045 fold) higher in $\mathrm{pCP}$ (mean $=5.66 \pm$ 0.39 ) compared to that of the aCP variant (mean $=1.40 \pm 0.25)$. 
negative. The results may indicate that there is no disease spectrum extending from RCC to $\mathrm{pCP}$.

Both pCP and RCC SE were immunoreactive for TREM-1. Moreover, TREM-1 was expressed in squamous metaplasia of cervicitis and lung cancer, while normal cervical SE and tongue carcinoma did not express TREM-1. The results indicated that TREM1 may be a novel marker of squamous metaplasia. The metaplastic theory posits that differentiated SE that forms part of the anterior pituitary or pituitary stalk undergoes metaplastic transformation to form pCP [10]. Thus, the fact that pCP epithelium was positive for TREM-1 was expected. Negative BRAF V600E immunoreactivity and positive TREM-1 immunoreactivity in RCC SE also support that there is no disease spectrum extending from RCC to $\mathrm{pCP}$ and the SE in RCC should be considered as metaplastic. Ogawa et al. demonstrated that patients with RCC presenting with significant squamous cell metaplasia had an extremely high risk (up to 50\%) of postoperative re-enlargement and often necessitated therapeutic reintervention [23]. Our results were similar to those of our previous study [21], while results from Buslei et al. indicate that the presence of squamous epithelial metaplasia or stratified SE in RCC is not a risk factor [22]. Duan et al. demonstrated that TREM-1 significantly promoted proliferation and invasion, but inhibited apoptosis of hepatocellular carcinoma (HCC) cells, which may be a prognostic factor for the clinical outcome of HCC [24]. Whether TREM-1 expression can explain why patients with RCC presenting with significant squamous metaplasia have an extremely high risk of recurrence should be further studied.

Several researchers presented evidence against such a rigid classification into aCPs and pCPs. Mixed type of craniopharyngioma was mainly defined as aCP with $\mathrm{SE}$ component. Petito et al. were among the first pathologists who questioned the validity of this distinction, after observing a high rate of lesions in a series of 245 CPs (approximately 33\%) presenting a mixture of both variants [8]. In a series of $131 \mathrm{CPs}$ reviewed by Szeifert et al., $38 \%$ of the cases belonged to the adamantinomatous group, $26 \%$ corresponded to the squamous epithelial type,

$\mathrm{a}$
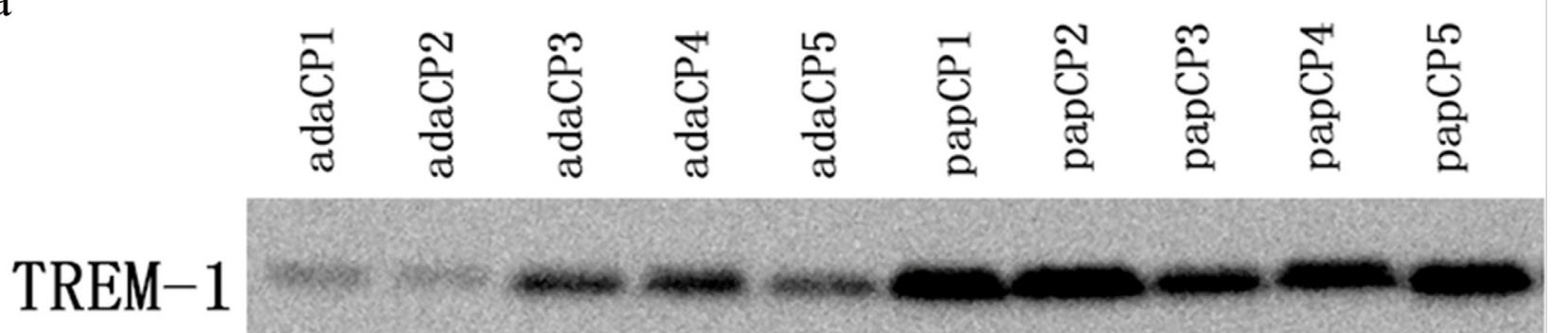

GAPDH

b

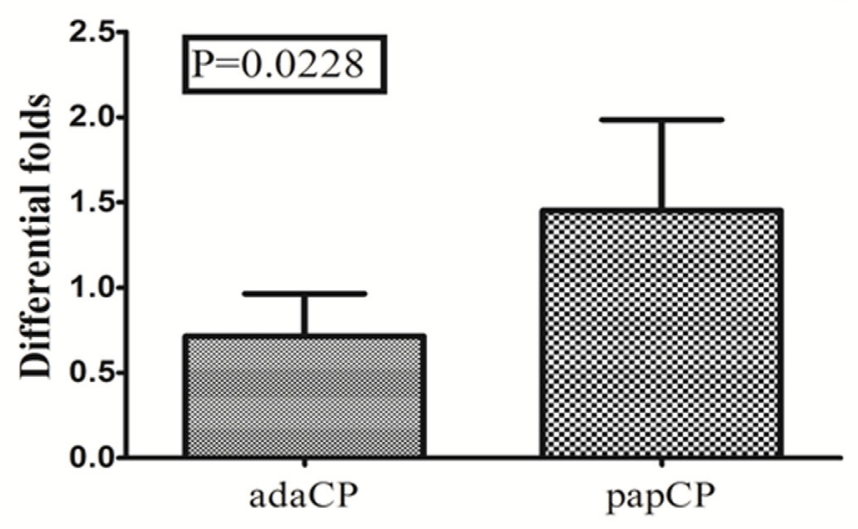

C

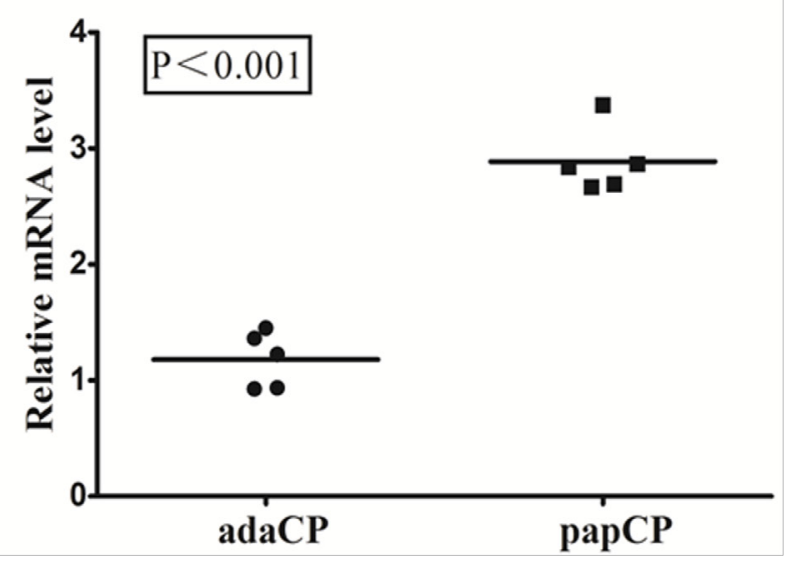

Figure 4: TREM-1 expression pattern in aCP and pCP tissues. a-b. Western blotting shows the protein level of TREM-1 in CP tissues. TREM-1 expression level is significantly ( $p=0.0228$; up to 1.89 fold) lower in aCP tissues ( $m e a n=0.714 \pm 0.112)$ compared to that of the $\mathrm{pCP}$ variant $(\mathrm{mean}=1.453 \pm 0.238)$. c. Evaluation of the relative TREM-1 expression revealed a significantly $(\mathrm{p}<0.001 ;$ up to 2.08 fold $)$ higher level in $\mathrm{pCP}($ mean $=2.99 \pm 0.12)$ compared to that of the $\mathrm{aCP}$ tissues $($ mean $=1.28 \pm 0.11)$ 
and $15 \%$ of the cases corresponded to a mixed variant combining features from both types [25]. In a series of 56 patients studied by Miller et al., three cases showed a mixed histological pattern [26]. Crotty et al. also reported 4 cases of mixed CPs in their seminal review of the squamous-papillary type; three of these cases exhibited alternating areas with definite adamantinomatous and papillary-squamous differentiation, while the epithelium displayed an intermingled appearance in the fourth lesion [27].
TREM-1 was also expressed in all SE of pCP and SE of RCC, while absent in RCC without SE. As the SE of aCP does not harbor V600e mutation, we concluded that the SE of aCP was of a different origin than the SE of pCP, indicating that a mixed type should not exist. Since $\mathrm{SE}$ in aCP, pCP, and RCC expresses TREM-1, we concluded that the SE may become metaplastic.

TREM-1 was expressed in all 5 mixed type CPs (aCP with SE), while, in pure aCP, TREM-1 was not expressed. In mixed type CPs, TREM-1 was only expressed in SE, while absent in adamantinomatous areas, including the

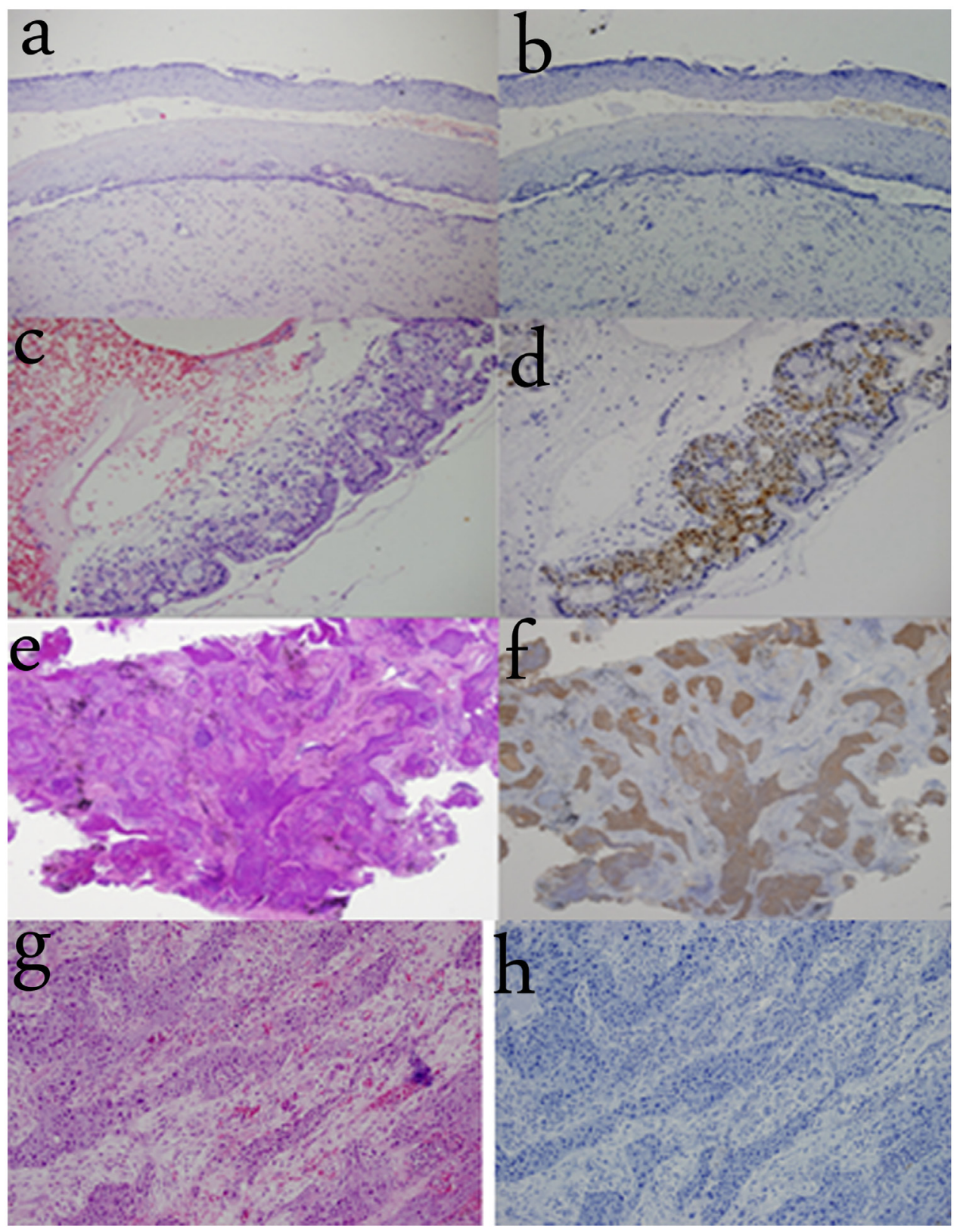

Figure 5: TREM-1 expression in other SE tissues. a-b. TREM-1 immunoreactivity is absent in normal cervical squamous epithelium. $(100 \times)$ c-d. TREM-1 immunoreactivity is observed in metaplastic cervical squamous epithelium and strongly detected in the cytoplasm and nuclei. $(200 \times)$ e-f. TREM-1 immunoreactivity is observed in squamous cell lung cancer (originating from metaplastic SE). $(40 \times)$ g-h. TREM-1 immunoreactivity is absent in tongue carcinoma (originating from SE). (200×) 
whorl-like cells and palisaded columnar epithelium. In all 5 "mixed" type CPs, V600E immunoreactivity was absent in SE and V600E mutation was not detected, indicating that SE in mixed type CP was not of the same origin as that in pCP. Thus, aCP with overlapping histological features of pCP should not be considered as a "mixed type" CP. In our previous study, inflammatory cell infiltration was common in aCP and may induce non-squamous epithelium to develop into squamous epithelial cells [28]. Positive TREM-1 immunoreactivity in SE of aCP suggests that SE in aCP may undergo metaplasia via inflammation.

Our additional findings that TREM-1 was only expressed in metaplastic SE, while absent in normal SE, suggest that TREM-1 is a novel marker of metaplasia. Based on our findings, RCC and pCP may represent two sides of a morphological spectrum, but seem to be distinct entities regarding their genetic make-up and, therefore, should be accurately differentiated. A variant showing overlapping histological features from aCP and $\mathrm{pCP}$ should not be considered as a mixed type. The SE in RCC and aCP may undergo metaplasia due to inflammation. However, there are some limitations in this study. First, the number of patients enrolled in this study may be too small to provide a strong conclusion. Secondly, the mechanisms involved in the genetic and molecular biological processes remain unknown. Thirdly, part of our study was similar to Buslei et al 's, however, Trem-1 expression pattern further supported Buslei et al 's result that RCC and pCP may represent two ends of a morphological spectrum. Future studies are warranted to determine the role played by TREM-1 in CP and RCC.

\section{MATERIALS AND METHODS}

\section{Patients}

Surgical specimens from 58 patients with CPs and RCCs were retrieved from the Neurosurgery Department of Nanfang hospital from January 2014 to December 2015. Each tumor sample was classified according to World Health Organization guidelines using hematoxylin and eosin (H\&E) as well as immunohistochemical staining (e.g., pan-cytokeratin, $\beta$-catenin, and V600E). Informed consent from each patient was obtained for all specimens for further scientific investigation, as approved by the local ethics committee of Southern Medical University.

\section{Cell culture}

In order to eliminate the influence of mesenchymal cells in CP tissues, we used cultured CP cells for our experiments. The CP specimens for tissue culture were minced into 1-mm fragments and cultured as previously reported [29-31]. We used the anchorage velocitydependent separation method to exclude mesenchymal cells and passage 3-cells were used for experiments.
The cultured CP cells were identified by assessing the expression of pan-CK. We also identified the cells by immunohistochemistry using V600E and $\beta$-catenin antibodies.

\section{Immunohistochemistry}

Surgical samples were prepared as previously described [32]. Briefly, paraffin-embedded sections were stained. Sections were cut at $4 \mu \mathrm{m}$. After deparaffinization and rehydration, the sections were heated for $20 \mathrm{~min}$ in sodium citrate buffer ( $\mathrm{pH}$ 6.0). For BRAF V600E, antigen retrieval was performed at $\mathrm{pH} 9$, using a microwave oven. Peroxidases were blocked by incubating the sections in $3 \%$ hydrogen peroxide for $10 \mathrm{~min}$. The sections were incubated overnight at $4{ }^{\circ} \mathrm{C}$ with a mouse monoclonal anti-BRAF V600E (dilution 1:100, Spring Bioscience Germany, E19290), rabbit polyclonal anti-CTNNB1 (dilution 1:100, ABclonal, Wuhan, China, A2064), rabbit polyclonal anti-TREM-1 (dilution 1:150, Sigma-Aldrich, St Louis, MO, USA, HPA 005563), and mouse monoclonal anti-pan cytokeratin (dilution 1:50, Abcam, Cambridge, MA, USA, AB7753). Following washing with PBS, the slides were incubated with the 2-step Dako REALTM EnVisionTM/HRP, Rabbit/Mouse (ENV) reagent (K-5007, Dako, Glostrup, Denmark). Visualization was achieved using DAB chromogen for 30-60 seconds. The sections were counterstained using hematoxylin, dehydrated, and then mounted using permount. In the negative controls, the primary antibody was replaced with PBS. Doubleimmunofluorescence staining was performed manually as described elsewhere [29] Cy2-anti-mouse and Cy3-antirabbit (1:1000; Life Technologies, Carlsbad, CA, USA) served as fluorescent secondary antibodies. Cell nuclei were stained with DAPI (Sigma-Aldrich) for $5 \mathrm{~min}$ at room temperature.

\section{Detection of TREM-1 in CP cells by western blot}

Cells were washed twice with $1 \times$ PBS and were then combined with $100 \mu \mathrm{L}$ of RIPA lysis buffer containing $1 \%$ PMSF protease inhibitor. Cells were lysed at $4^{\circ} \mathrm{C}$ for 20 min and the solution was centrifuged at $12,000 \mathrm{rpm}$ for $30 \mathrm{~min}$. The supernatant was collected for protein assay using the bicinchoninic acid (BCA) method. Proteins were separated using $10 \%$ sodium dodecyl sulfate polyacrylamide gel electrophoresis and transferred to a polyvinylidene difluoride membrane. After blocking, the membrane was incubated at $4^{\circ} \mathrm{C}$ overnight with rabbit polyclonal anti-TREM-1 (dilution 1:1200, Sigma-Aldrich, HPA 005563). After washing, the secondary antibody, anti-rabbit-HRP, was used. Bands were visualized using an enhanced-chemiluminescence kit (Amersham Pharmacia Biotechnology Inc., Piscataway, NJ, USA). An antiGAPDH antibody (dilution 1:1000, KeyGEN BioTECH, 
Jiangsu, China, KGAA002-2) was used as a loading control. Each experiment was performed in triplicate.

\section{Quantitative reverse transcription PCR}

Total RNA of cultured aCP and pCP cells was isolated with Trizol Reagent (TaKaRa, Tokyo, Japan) according to manufacturer's protocol. After digestion with RNase-free DNase I (TaKaRa), the total amount of RNA was determined by measuring probes on a NanoDrop (Thermo Fisher Scientific, Waltham, MA, USA), followed by reverse transcription using SuperScript First-Strand Synthesis System (Invitrogen, Carlsbad, CA, USA) with oligo (dT) primers. qRT-PCR with Sybr Green II (Applied Biosystems, Santa Clara, CA, USA) was employed to quantitatively assess the expression of TREM-1 in $6 \mathrm{aCP}$ and $6 \mathrm{pCP}$ cultured cells. All analyses were carried out with the Applied Biosystems 7500 Fast Real-Time-PCR. $\beta$-actin was used as an endogenous control for cDNA amount.

Primers for human TREM-1 were as follows: forward, 5'-GGCCACACCAACCTTCTG-3 and reverse, 5'-AGTGCCTGCCTCAATGTCTCCA-3'. Primers for actin were as follows: forward, 5'-AGAAAATCTGGCACCACACC-3' and reverse, 5'-AGAGGCGTACAGGGATAGCA-3'. Analysis was conducted using the $2^{-\Delta \Delta C T}$ method according to the manufacturer's instructions (Applied Biosystems). All analyses were carried out in quadruplicate and evaluated statistically.

\section{Mutation analysis of BRAF V600E and beta- catenin (CTNNB1 exon 3)}

Tumor genomic DNA from each formalin-fixed and paraffin-embedded slide was extracted with the Maxwell system (Promega, Madison, WI, USA). The mutation status was analyzed with the 70plex liquidchip platform (Surexam, Guangzhou, China) for the 70 alleles. The 70plex includes five major steps: (1) multiplex PCR to amplify 70 target genes; (2) exonuclease I and shrimp alkaline phosphatase (EXOSAP) cleaning to remove excess nucleotides and primers; (3) allele specific primer extension where EXO-SAP-cleaned PCR products were amplified with 70 specific primers that were linked to 70 universal tags; (4) hybridization to beads, and (5) Luminex analysis and the median fluorescence intensity was read and analyzed. As part of quality control, samples were randomly sent to independent companies for DNA sequencing analysis.

\section{Statistics}

Statistical analyses were performed using SPSS statistical software for Windows version 19.0 (SPSS, Chicago, IL, USA). A p value $<0.05$ was considered statistically significant. When the samples came from a normally distributed population, an unpaired Student $t$-test was conducted to resolve hypothesized differences.

\section{ACKNOWLEDGMENTS}

We thank Fan Jun, Lu Yuntao, and Qiu Binghui for their excellent technical support and patient care. This work was funded by the President Foundation of Nanfang Hospital, Southern Medical University, project \# 2015C018.

\section{CONFLICTS OF INTEREST}

The authors declare that they have no conflicts of interest.

\section{REFERENCES}

1. Larkin SJ, Ansorge O. Pathology and pathogenesis of craniopharyngiomas. Pituitary. 2013; 16:9-17.

2. Martinez-Barbera JP, Buslei R. Adamantinomatous craniopharyngioma: pathology, molecular genetics and mouse models. J Pediatr Endocrinol Metab. 2015; 28:7-17.

3. Prabhu VC, Brown HG. The pathogenesis of craniopharyngiomas. Childs Nerv Syst. 2005; 21:622-627.

4. Zada G, Lin N, Ojerholm E, Ramkissoon S, Laws ER. Craniopharyngioma and other cystic epithelial lesions of the sellar region: a review of clinical, imaging, and histopathological relationships. Neurosurg Focus. 2010; 28:E4.

5. Okada T, Fujitsu K, Ichikawa T, Mukaihara S, Miyahara K, Kaku S, Uryuu Y, Niino H, Yagishita S, Shiina T. Coexistence of adamantinomatous and squamous-papillary type craniopharyngioma: case report and discussion of etiology and pathology. Neuropathology. 2012; 32:171-173.

6. Prieto R, Pascual JM. Craniopharyngiomas with a mixed histological pattern: the missing link to the intriguing pathogenesis of adamantinomatous and squamous-papillary varieties? Neuropathology. 2013; 33:682-686.

7. Weiner HL, Wisoff JH, Rosenberg ME, Kupersmith MJ, Cohen H, Zagzag D, Shiminski-Maher T, Flamm ES, Epstein FJ, Miller DC. Craniopharyngiomas: a clinicopathological analysis of factors predictive of recurrence and functional outcome. Neurosurgery. 1994; 35:1001-1010, 1010-1011.

8. Petito CK, DeGirolami U, Earle KM. Craniopharyngiomas: a clinical and pathological review. Cancer. 1976; 37:1944-1952.

9. Wolfe SQ, Heros RC. A Rathke cleft cyst to craniopharyngioma: is there a spectrum? J Neurosurg. 2010; 112:1322-1323, 1323.

10. Mori M, Takeshima H, Kuratsu J. Expression of interleukin-6 in human craniopharyngiomas: a possible 
inducer of tumor-associated inflammation. Int J Mol Med. 2004; 14:505-509.

11. Pettorini BL, Inzitari R, Massimi L, Tamburrini G, Caldarelli M, Fanali C, Cabras T, Messana I, Castagnola $\mathrm{M}$, Di Rocco C. The role of inflammation in the genesis of the cystic component of craniopharyngiomas. Childs Nerv Syst. 2010; 26:1779-1784.

12. Andoniadou CL, Gaston-Massuet C, Reddy R, Schneider RP, Blasco MA, Le Tissier P, Jacques TS, Pevny LH, Dattani MT, Martinez-Barbera JP. Identification of novel pathways involved in the pathogenesis of human adamantinomatous craniopharyngioma. Acta Neuropathol. 2012; 124:259-271.

13. Buslei R, Nolde M, Hofmann B, Meissner S, Eyupoglu IY, Siebzehnrubl F, Hahnen E, Kreutzer J, Fahlbusch R. Common mutations of beta-catenin in adamantinomatous craniopharyngiomas but not in other tumours originating from the sellar region. Acta Neuropathol. 2005; 109:589-597.

14. Campanini ML, Colli LM, Paixao BM, Cabral TP, Amaral FC, Machado HR, Neder LS, Saggioro F, Moreira AC, Antonini SR, de Castro M. CTNNB1 gene mutations, pituitary transcription factors, and MicroRNA expression involvement in the pathogenesis of adamantinomatous craniopharyngiomas. Horm Cancer. 2010; 1:187-196.

15. Larkin SJ, Preda V, Karavitaki N, Grossman A, Ansorge O. BRAF V600E mutations are characteristic for papillary craniopharyngioma and may coexist with CTNNB1mutated adamantinomatous craniopharyngioma. Acta Neuropathol. 2014; 127:927-929.

16. Brastianos PK, Taylor-Weiner A, Manley PE, Jones RT, Dias-Santagata D, Thorner AR, Lawrence MS, Rodriguez FJ, Bernardo LA, Schubert L, Sunkavalli A, Shillingford N, Calicchio ML, Lidov HG, Taha H, Martinez-Lage M, et al. Exome sequencing identifies BRAF mutations in papillary craniopharyngiomas. Nat Genet. 2014; 46:161-165.

17. Holsken A, Sill M, Merkle J, Schweizer L, Buchfelder M, Flitsch J, Fahlbusch R, Metzler M, Kool M, Pfister SM, von Deimling A, Capper D, Jones DT, Buslei R. Adamantinomatous and papillary craniopharyngiomas are characterized by distinct epigenomic as well as mutational and transcriptomic profiles. Acta Neuropathol Commun. 2016; 4:20.

18. Ford JW, McVicar DW. TREM and TREM-like receptors in inflammation and disease. Curr Opin Immunol. 2009; 21:38-46.

19. Klesney-Tait J, Turnbull IR, Colonna M. The TREM receptor family and signal integration. Nat Immunol. 2006; 7:1266-1273.

20. Zhou J, Qi ST, Chen LG, Huang CR, You J, Zhang C, Pan J. [Expression pattern of inflammatory cytokines at various inflammatory levels of adamantinomatous craniopharyngioma]. [Article in Chinese]. Zhonghua Yi Xue Za Zhi. 2013; 93:2499-2501.

21. Chotai S, Liu Y, Pan J, Qi S. Characteristics of Rathke's cleft cyst based on cyst location with a primary focus on recurrence after resection. J Neurosurg. 2015; 122:1380-1389.

22. Schweizer L, Capper D, Holsken A, Fahlbusch R, Flitsch J, Buchfelder M, Herold-Mende C, von Deimling A, Buslei R. BRAF V600E analysis for the differentiation of papillary craniopharyngiomas and Rathke's cleft cysts. Neuropathol Appl Neurobiol. 2015; 41:733-742.

23. Ogawa Y, Watanabe M, Tominaga T. Prognostic factors of operated Rathke's cleft cysts with special reference to re-accumulation and recommended surgical strategy. Acta Neurochir (Wien). 2011; 153:2427-2433, 2433.

24. Duan M, Wang ZC, Wang XY, Shi JY, Yang LX, Ding ZB, Gao Q, Zhou J, Fan J. TREM-1, an inflammatory modulator, is expressed in hepatocellular carcinoma cells and significantly promotes tumor progression. Ann Surg Oncol. 2015; 22:3121-3129.

25. Szeifert GT, Sipos L, Horvath M, Sarker MH, Major O, Salomvary B, Czirjak S, Balint K, Slowik F, Kolonics L, Et A. Pathological characteristics of surgically removed craniopharyngiomas: analysis of 131 cases. Acta Neurochir (Wien). 1993; 124:139-143.

26. Miller DC. Pathology of craniopharyngiomas: clinical import of pathological findings. Pediatr Neurosurg. 1994; 21:11-17.

27. Crotty TB, Scheithauer BW, Young WJ, Davis DH, Shaw EG, Miller GM, Burger PC. Papillary craniopharyngioma: a clinicopathological study of 48 cases. J Neurosurg. 1995; $83: 206-214$.

28. Qi ST, Zhou J, Pan J, Zhang C, Silky C, Yan XR. Epithelialmesenchymal transition and clinicopathological correlation in craniopharyngioma. Histopathology. 2012; 61:711-725.

29. Liszczak T, Richardson EJ, Phillips JP, Jacobson S, Kornblith PL. Morphological, biochemical, ultrastructural, tissue culture and clinical observations of typical and aggressive craniopharyngiomas. Acta Neuropathol. 1978; 43:191-203.

30. Yoshida J, Kobayashi T, Kageyama N, Kanzaki M. Symptomatic Rathke's cleft cyst. Morphological study with light and electron microscopy and tissue culture. J Neurosurg. 1977; 47:451-458.

31. Chen M, Zheng SH, Liu Y, Shi J, Qi ST. Periostin activates pathways involved in epithelial-mesenchymal transition in adamantinomatous craniopharyngioma. J Neurol Sci. 2016; 360:49-54.

32. Frei K, Gramatzki D, Tritschler I, Schroeder JJ, Espinoza L, Rushing EJ, Weller M. Transforming growth factor-beta pathway activity in glioblastoma. Oncotarget. 2015; 6:59635977. doi: 10.18632/oncotarget.3467. 